\title{
CYTOPATHOLOGICAL STUDIES ON CANINE MAMMARY TUMORS OF EPITHELIAL ORIGIN
}

\author{
Y. M. KOTRAPPA, S. RAO ${ }^{1}$, B. C. GIRISH ${ }^{2 *}$ \\ AND S. M. BYREGOWDA ${ }^{3}$ \\ Syngene International Ltd, Biocon Park \\ Jigani Link Road, Bommasandra, Bengaluru \\ Karnataka- 560 099, India
}

\begin{abstract}
Canine mammary tumors are considered as useful animal models in understanding human breast cancers. They can present either as malignant or benign variants and can have different origin from epithelial to mesenchymal tissues. In the current investigation, based on histogenesis, the mammary tumors were classified as adenocarcinoma, co-existing adenocarcinoma and squamous cell carcinoma, malignant mixed mammary tumor and fibroadenoma. Cytologically, the epithelial cells were arranged in sheets, clusters and also as individual cells with occasional acinar structures having eccentrically placed nuclei in adenocarcinomas. In the coexisting type with squamous cell carcinoma features of adenocarcinoma and squamous cell carcinomas were noticed. The cytology of malignant mixed mammary gland tumor revealed epithelial as well as mesenchymal cells. Cytological smears from a solitary case of fibroadenoma revealed less number of cells that exhibited uniform size with eccentrically placed nucleus.
\end{abstract}

Key words: Adenocarcinoma, Canine, Cytology, Mammary tumors

Canine mammary tumors are often considered useful animal models in understanding the pathobiology of human breast cancers. The scientific community under the new concept of one health can hugely benefit taking the advantage of this interdisciplinary data. Detection of new biomarkers is useful in earlier diagnosis and also to arrive at better therapeutic interventions. It has been documented widely that mammary tumors of dogs have large number of similarities with the human counterparts. These similarities range from epidemiological data to the histological presentation of the neoplastic lesions (Misdorp and Hart, 1976; Queiroga et al., 2011; Girish et al., 2011).

\footnotetext{
*Corresponding Author

${ }^{1}$ Department of Pathology, Veterinary College, Hebbal, Bengaluru. Karnataka, 560024, India

${ }^{2}$ Department of Pathology, Veterinary College, Gokula, Vidyanagara-Post, Hassan, 573202, India

${ }^{3}$ Institute of Animal Health and Veterinary Biologicals, Bengaluru. Karnataka, 560024, India
} 
Canine mammary gland tumors can present either as malignant or benign variants. They can have different tissue origin from epithelial or glandular tissues to mesenchymal or connective tissues. Majority of the malignant types are known to fall under classification of epithelial tumors (Monlux et al., 1977; Misdorp, 1999). Skin and mammary tumors make up the two largest groups of neoplasms in dogs. Their incidence was found to be $33.4 \%$ and $36 \%$ of cases, respectively (Sanja et al., 2005). In another study, mammary tumors were found to account for approximately $50 \%$ of all neoplasms in female dogs (Brody et al., 1985).

As mammary tumors are one of the major animal health problems that are increasing day by day, need for further advancement of veterinary oncology has been emphasized in the recent past (Yaritza et al., 2015). Routinely used histological methods require invasive sample collection and long processing duration. Hence, necessity for quick and low-cost method of cancer diagnosis has been felt by the veterinary practitioners. Cytology fulfills this by being an inexpensive, rapid, repeatable and less painful technique. Cytology refers to microscopic evaluation of cells that have either exfoliated naturally or has been clinically detached from the body/organ/ tissue mass (Haziroglu et al., 2010). Though this diagnostic method is fully exploited in human medicine, the same has to catch up in veterinary practice (Haziroglu et al., 2010). Recently, with the inclusion of tumor immune markers (Immunocytochemistry) that can be used on cytology smears, the sensitivity and specificity of cytological diagnosis has been increased further.

Mammary tumors can be classified in different ways. Few of those criteria include, tissue of origin, whether they are benign or malignant, based on cellular differentiation, staging and grading. Tissues of origin may include glandular (adenoma/ adenocarcinoma), ductular (papilloma/ carcinoma), myoepithelial and pluripotential (mixed) cells. However, some ambiguity about the histological origin of many mammary tumors which present themselves as undifferentiated ones and involve many tissue types remains (Gerry, 2009).

The current investigation was aimed to determine and describe the clinical, gross pathological, cytological, and microscopic pathology of mammary tumors of epithelial origin in dogs. Other neoplasms of nonepithelial origin are not discussed here.

\section{MATERIALS AND METHODS}

The investigation was carried out in the Department of Veterinary Pathology, Veterinary College, Bengaluru. The dogs presented to college hospitals, other nearby private clinics with the history of tumor growths and post mortems formed the source for the present investigation. Upon presentation of the case, detailed information of the patients covering breed, age, sex, color, clinical findings 
shown by the animals such as shape, size, color and location of the growths were recorded. For cytological evaluation, different methods were used to collect tissue samples based on the nature of the growths. Fine needle aspiration cytology (FNAC) and fine needle aspiration biopsy (FNAB) were employed for superficial nodular growths. Impression smears and scraping smears were used for neoplasms with ulcers. Scraping method was used for surgically excised growths.

The smears were stained using a battery of cytological stains. It included standard Giemsa, New Methylene blue, Toluidine blue, and Papanicolaou staining techniques (Luna, 1968). Criteria for malignancy were adopted (Villiers and Dunn, 1998; Kotrappa et al., 2014) and cellular, cytoplasmic and nuclear details were described objectively.

Tissue samples collected were fixed immediately in $10 \%$ Neutral Buffered Formalin. Representative samples from the neoplastic growths were processed by routine paraffin embedding technique. Sections of five micron thickness were cut using Erma microtome with disposable blades. These sections were then stained with routine hematoxylin and eosin method (Luna, 1968). Special stains such as Van Gieson's (connective tissue and keratin), Masson's trichrome (connective tissue and keratin) and Toluidine blue (mast cell granules) were used to aid in further characterization.

\section{RESULTS}

In the present study, 15 mammary tumors were recorded out of 67 cases. The age of the animals ranged from 6 to 15 years with an average age of 9.8 years and all occurred in female dogs. The breeds involved were nondescript (5), Pomeranian (4), Labrador (3), Lhasa apso (1), Cocker spaniel (1) and German Shepherd (1). Based on histogenesis, the mammary tumors were classified as adenocarcinoma, co-existing adenocarcinoma and squamous cell carcinoma, malignant mixed mammary tumor and fibroadenoma.

Adenocarcinoma: Out of 67 cases, 7 were adenocarcinomas of mammary gland. Age of the animals varied from 6 to 15 years with an average age of 9.57 years. Out of seven affected dogs 4 were intact and 3 were neutered. The breeds involved were Pomeranian (2), Lhasa apso (1), Cocker spaniel (1), German Shepherd (1), Labrador (1) and nondescript (1). Grossly, all the adenocarcinomas were firm and hard in consistency. The tumor masses showed lobulations and measured 4 to $8 \mathrm{~cm}$ in diameter. The growths involved inguinal pair (3) thoracic pair (3) and both thoracic and inguinal pairs (1) of mammary gland. Cytologically the epithelial cells were arranged in sheets, clusters and also as individual cells with occasional acinar structures having eccentrically placed nuclei. The cells revealed moderate to 
marked anisocytosis and anisokaryosis. They were round, oval or caudate shaped with spherical oval or irregular nuclei. The nuclei were moderate to intensely stained with coarse chromatin and prominent nucleoli in some cells.

Histopathologically, papillary adenocarcinoma was characterized by predominantly papillary arrangement with tubular and papillary structures along with cystic spaces lined by cuboidal to columnar epithelial cells (Fig. 1).

\section{Co-existing adenocarcinoma and} squamous cell carcinoma: Three cases of adenocarcinoma along with squamous cell carcinoma were recorded out of 15 mammary tumors investigated. The age of the animals ranged between 8 to 14 years with a mean age of 10.66 years. All the cases were encountered in intact females. The tumor masses were located, one each in inguinal and thoracic and one in both thoracic and abdominal mammary glands.

Grossly, the size of coexisting growths ranged from 4 to $8 \mathrm{~cm}$ at their greatest diameters. All were firm and hard in consistency. The masses were irregularly lobulated with areas of necrosis and hemorrhages. Few cystic areas filled with brownish colored fluid were also evident on the cut surface of all the growths. One of the growths was severely ulcerated with oozing of brownish colored fluid.

The cytological smears revealed high cellularity and comprised both glandular and squamous epithelial cells (Fig. 2). The cytological details of malignant cells of adenocarcinoma and squamous cells carcinoma were similar to coexisting adenocarcinoma and squamous cell carcinoma of mammary gland.

Microscopically, features of both adenocarcinoma and squamous cell carcinomas were noticed and the growths existed as two separate entities (Fig. 3). A case of co-existing adenocarcinoma and squamous cell carcinoma of mammary gland was found to be metastatic to liver and pancreas.

\section{Malignant mixed mammary tumor:} Four cases were malignant mixed tumors out of 15 mammary tumors encountered. The age of the affected animals varied from 8 to 11 years with a mean age of 10 years. The location of the tumor masses was inguinal (1), abdominal (1), thoracic and inguinal (1), thoracic and abdominal (1) mammary glands and all occurred in intact females.

Grossly, the size of the tumor masses varied from 1 to $8 \mathrm{~cm}$ at their greatest diameter. All the growths were firm and hard in consistency. All were irregular in shape with multilobulations. Two cases were ulcerated with areas of necrosis. The cut surface was pinkish white colored in all cases.

The cytology of malignant mixed mammary gland tumor revealed epithelial 
Indian Journal of Animal Health, June, 2018

Studies on canine mammary tumors

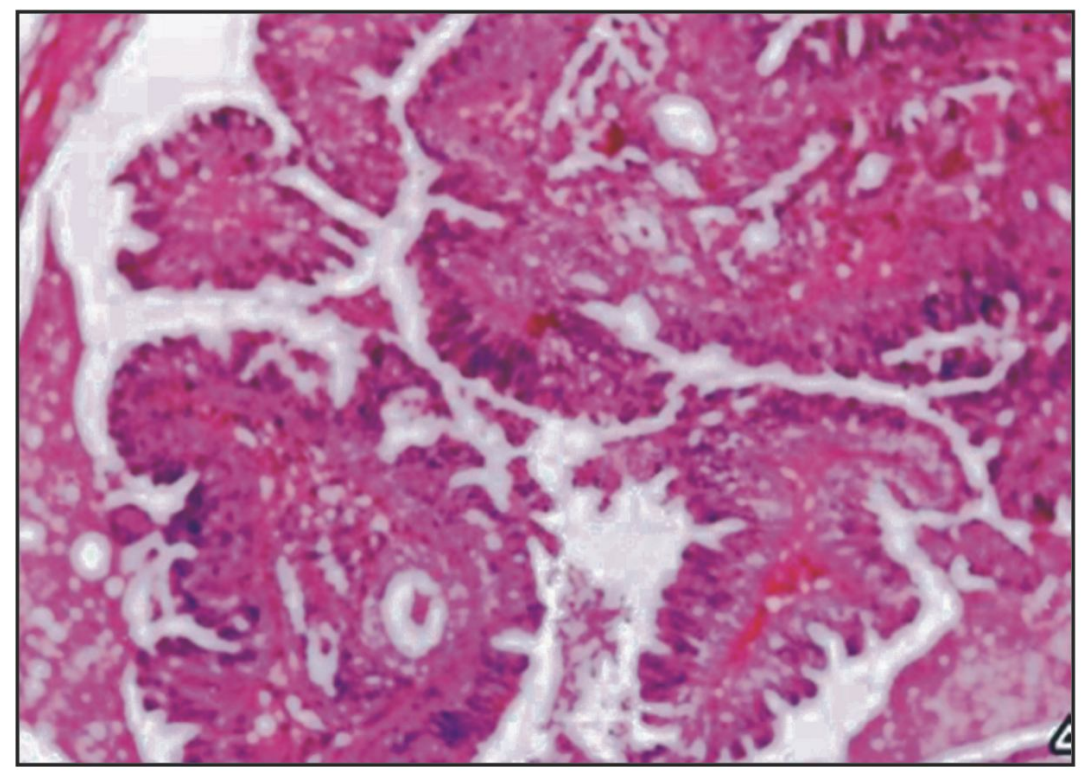

Fig. 1. Section of adenocarcinoma of mammary gland showing papillary arrangement of proliferating cells with occasional duct like structures, (H\&E X 12.5)

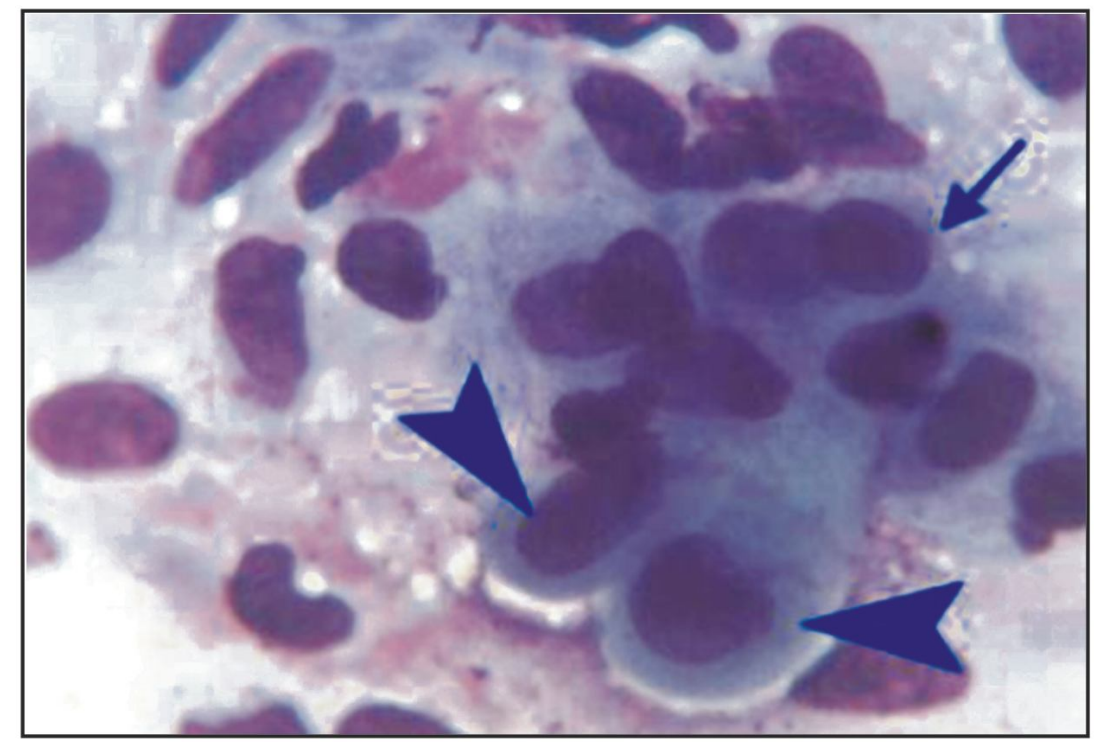

Fig. 2. Cytological smear of co-existing adenocarcinoma and squamous cell carcinoma of mammary gland showing malignant squamous (arrow head) and glandular epithelial cells (arrow), (Giemsa $X$ 125) 
Indian Journal of Animal Health, June, 2018

Studies on canine mammary tumors

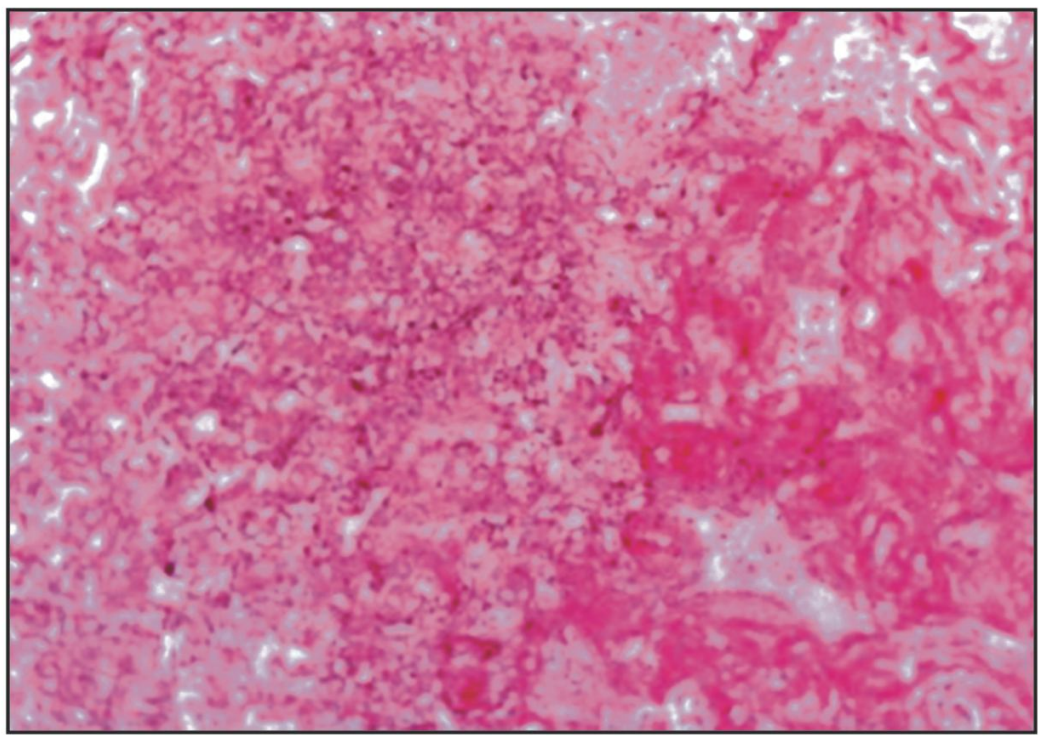

Fig. 3. Section of co-existing adenocarcinoma and squamous cell carcinoma showing glandular arrangement of proliferating cells of adenocarcinomas (Left) and keratin component of squamous cell carcinoma (Right), (H\&E X 12.5)

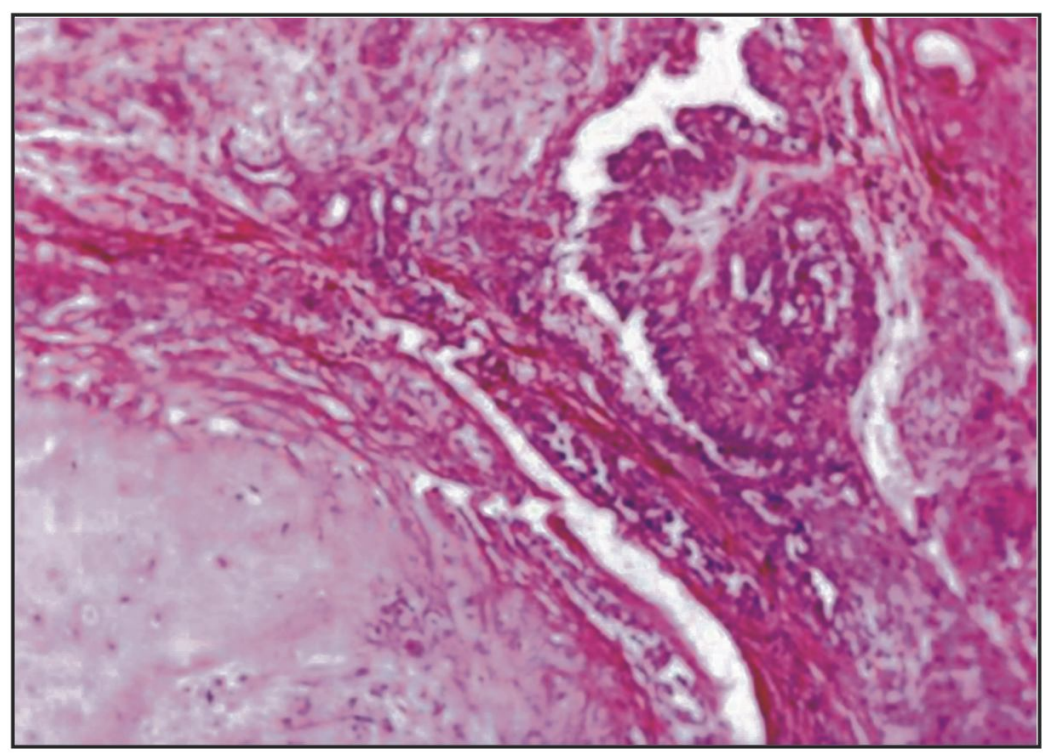

Fig. 4. Section of malignant mixed tumor of mammary gland showing malignant epithelial and mesenchymal osseous components, (H \& E X 12.5) 
as well as mesenchymal cells. The malignant epithelial cells occurred in sheets and showed the similar characteristics of cells of adenocarcinoma.

Histopathologically, the tumor masses were composed of both epithelial and connective tissue components (Fig. 4). The neoplastic epithelial component comprised tubular, acinar structures lined by cuboidal to columnar type of cells with pleomorphism and numerous mitotic figures.

Fibroadenoma : A single case of fibroadenoma was recorded in 8 year old female intact Labrador breed, involving the thoracic mammary gland. Grossly, the size of the tumor mass was $8 \times 8 \times 7 \mathrm{~cm}$ with approximate weight of one $\mathrm{Kg}$ and was found attached to the sternal region. The tumor was soft, lobulated and encapsulated.

The cytological smears revealed less number of cells. The cells were of uniform size with moderate amount of cytoplasm and eccentrically placed nucleus. In addition to epithelial cells, elongated basophilic cells containing oval condensed nuclei with cytoplasmic extension from both the ends were also observed. Microscopically it revealed moderate to heavy stromal connective tissue proliferation around the neoplastic tubular structures. Hyalinization of connective tissue with a few areas of calcification and infiltration of lymphocytes and plasma cells were also seen.

\section{DISCUSSION}

In the current investigation, 15 mammary tumors were recorded out of 67 cases. The age of the animals ranged from 6 to 15 years with an average age of 9.8 years.

All the malignant mammary tumors in the present study occurred as single, firm, well-circumscribed masses to multiple infiltrative nodules involving one or more glands as observed earlier (Allen et al., 1986; Maddux and Shull, 1993; Shekar et al., 2000). However, fibroadenoma a benign tumor encountered in the present study was a huge, soft, lobulated growth weighing around $1 \mathrm{Kg}$ and found attached to the sternal region.

Adenocarcinoma: In the study seven adenocarcinomas of mammary gland were recorded. Cytologically, the cells were arranged in sheets/clusters/as individual cells with occasional acinar structures with eccentrically placed nuclei and marked anisocytosis and anisokaryosis. The cytological findings well tallied with the previous descriptions (Maddux and Shull, 1993; Henson, 2001). Occasional spindle shaped cells having elongated dense nuclei were also noticed in the present study, which could be myoepithelial cells. All types of adenocarcinomas encountered in the 
present study, such as papillary, tubular, mucinous, schirrous and solid type presented similar cytological picture.

Histopathologically, papillary adenocarcinoma was characterized by predominantly papillary arrangement with tubular and papillary structures. Tubular and papillary adenocarcinoma was characterized by presence of both papillary and tubular structures with myoepithelial cell proliferation. Mucinous adenocarcinoma was composed of acinar structures with plenty of mucinous secretion within the lumen. Proliferating myoepithelial cells were in the form of spindle shaped interstitial cells dispersed in mucinous ground substance, with occasional whorled arrangement of myoepithelial cells. Schirrous adenocarcinoma revealed both secretory and myoepithelial component with abundant collagenous stromal proliferation. Solid carcinomas were composed of sheets of proliferating neoplastic epithelial cells with occasional acinar structures. These findings tallied well with the observations of earlier workers (Hampe and Misdrop, 1974; Moulton et al., 1986; Shekar et al., 2000).

Further, mild to moderate infiltration of lymphocytes, plasma cells and pigment containing macrophages in the surrounding connective tissue and occasionally within the tumor growth, which was also observed in the present investigation, was attributed to the host tumor immune response (Gilbertson et al., 1983).

Co-existing adenocarcinoma and squamous cell carcinoma: Three cases of adenocarcinoma along with squamous cell carcinoma were recorded. The masses were irregularly lobulated with areas of necrosis and hemorrhages. The cytological smears revealed high cellularity and comprised both glandular and squamous epithelial cells. The tumor masses revealed proliferating neoplastic epithelial cells forming glandular structures with eosinophilic material in their lumen and adjacent solid cords of proliferating neoplastic cells with lamellated keratin at many areas and individual cell keratinization. Multifocal areas of necrosis and inflammatory reactions were also noticed. The histology of the individual tumors tallied well with the observations of earlier workers (Hampe and Misdrop, 1974; Shekar et al., 2000).

Malignant mixed mammary tumor: Four malignant mixed tumors cases were recorded in the study. All the growths were firm and hard in consistency with irregular in shape with multilobulations. Cytologically they revealed epithelial as well as mesenchymal cells. Spindle shaped cells consisting of plumpy elongated nuclei with variable chromicity, binucleation, and basophilic cytoplasm indicative of mesenchymal cells 
(chondrocytes) were also seen. These findings were in conformity with those reported by Allen et al.,(1986) and Henson, (2001). The tumor masses were composed of both epithelial and connective tissue components. The neoplastic epithelial component comprised tubular, acinar structures with numerous mitotic figures. The mesenchymal component comprised of cartilaginous and osseous tissues. These findings were in tune with those described earlier (Hampe and Misdrop, 1974; Yamagami et al., 1996).

Fibroadenoma: One case of fibroadenoma out of 15 mammary tumors was recorded in 8 year old female intact Labrador breed, involving the thoracic mammary gland. Grossly, the size of the tumor mass was $8 \times 8 \times 7 \mathrm{~cm}$ with approximate weight of one $\mathrm{Kg}$ and was found attached to the sternal region. The tumor was soft, lobulated and encapsulated. Cytologically, the cells

\section{REFERENCES}

Allen WS, Prasse KW and Mahaffey AE, 1986. Cytologic differentiation of benign from malignant mammary tumors. Vet Pathol, 23: 649-655

Brody RS, Goldschmidt MH and Roszel JR, 1985. Canine mammary gland neoplasia. J Am Anim Hosp Assoc, 19: 61-90

Gerry P, 2009. Mammary tumors in dogs. Irish Vet J, 62(1): 50-56

Gilbertson SR, Kurzman JD, Zachrau RE, Hurity AI and Black MM, 1983. Canine were of uniform size with moderate amount of cytoplasm. Elongated basophilic cells containing oval condensed nuclei with cytoplasmic extension from both the ends were also observed. These findings were in conformity with the previous reports (Allen et al., 1986; Henson, 2001). Histopathology of fibroadenoma revealed moderate to heavy stromal connective tissue proliferation around the neoplastic tubular structures. These findings were in accordance with the previous reports (Hampe and Misdrop, 1974; Shekar et al., 2000).

In summary, the mammary tumors of dogs with histogenesis of epithelial type were described and classified considering their gross, cytological and histopathological features. These cytopathological observations may be useful in studying canine tumors as effective animal models for human breast cancers in the near future.

mammary epithelial neoplasms: Biologic implications of morphologic characteristics assessed in $232 \mathrm{dogs}$. Vet Pathol, 20: 127-142

Girish BC, Suguna R, Byregowda SM and Kotrappa YM, 2011. Canine squamous cell carcinoma: a review of 17 cases. Braz J Vet Pathol, 4(2): 79-86

Hampe JF and Misdrop W, 1974. Tumours and dysplasias of the mammary gland. Bull Wld Hlth Org, 50: 111-133 
Haziroglu R, Yardimci B, Aslan S, Yildirim MZ and Yumusak N et al., 2010. Cytological evaluation of canine mammary tumours with fine needle aspiration biopsy technique. Revue Méd Vét, 161(5): 212218

Henson KL, 2001. Reproductive system. In: Atlas of Canine and Feline Cytology (Raskin RE, Meyer DJ, eds). W. B. Saunders Co., Philadelphia, pp 277-312

Kotrappa YM, Suguna R, Girish BC, Byregowda SM and Manjunatha $\mathrm{K}$, 2014. Cytological and histopathological studies of mesenchymal and melonotic neoplasms in dogs. J Cell Tissue Res, 14(3): 4477-4483

Luna AG, 1968. In, Manual of histology and special staining technique. Armed Force Institute of Pathology, Magraw Hill Book Co., London

Maddux JM and Shull RM, 1993. Subcutaneous glandular tissue: mammary, salivary, thyroid and parathyroid. In: Diagnostic Cytology of the Dog and Cat (Cowell RL, Tyler RD, eds). American Veterinary Pub. Inc., California, pp 83-92

Misdorp W, 1999. Histological classification of mammary tumors of the dog and the cat. Washington: Armed Forces Institute of Pathology in cooperation with the American Registry of Pathology and the World Health Organization Collaborating Center for Worldwide Reference on Comparative Oncology, pp 59

Misdorp W and Hart AA, 1976. Prognostic factors in canine mammary cancer. J Natl Cancer Inst, 56: 779-786
Monlux W, Roszel JF, Macveana DW and Palmer TW, 1977. Classification of epithelial canine mammary tumors in a defined population. Vet Pathol, 14: 194217

Moulton JE, Rosenblatt LS and Goldman M, 1986. Mammary tumours in a colony of Beagle dogs. Vet Pathol, 23: 741-749

Queiroga FL, Raposo T, Carvalho MI, Prada J and Pires I, 2011. Canine mammary tumours as a model to study human breast cancer: most recent findings. In Vivo, 25: $455-465$

Sanja AK, Kukolj V, Marinkovic D and Knezevic M, 2005. Retrospective study of canine epithelial and melanocytic tumors. Acta Veterinaria (Beograd), 55(4), 319326

Shekar CS, Vijayasarathi SK, Sreenivas Gowda RN, Satyanarayana ML and Suguna R, 2000. The role of myoepithelial cells in canine mammary tumours. Indian J Anim Sci, 70: 12331235

Villiers EJ and Dunn JK, 1998. Collection and preparation of smears for cytological examination. In Pract, 20: 370-377

Yamagami T, Kobayashi T, Takahashi K and Sugiyama, 1996. Prognosis for canine malignant mammary tumours based on TNM and histologic classification. J Vet Med Sci, 58: 1078-1083

Yaritza S, Adelys M, Daniel D and Laura R, 2015. Epidemiological study of mammary tumors in female dogs diagnosed during the period 2002-2012: A Growing Animal Health Problem. PLOS ONE, 1:1-15 